\title{
Integrating Rapid Diagnostics and Antimicrobial Stewardship in Two Community Hospitals Improved Process Measures and Antibiotic Adjustment Time
}

\author{
Ashley M. Lockwood, PharmD; ${ }^{1}$ Katherine K. Perez, PharmD; ${ }^{1,2}$ William L. Musick, PharmD ${ }^{1}{ }^{J}$ udy O. Ikwuagwu, \\ PharmD $;{ }^{1}$ Engie Attia, PharmD $;^{1}$ Oyejoke O. Fasoranti, PharmD ${ }^{1}$ Patricia L. Cernoch, MT; ${ }^{1}$ Randall J. Olsen, MD, PhD; ${ }^{1,2}$ \\ James M. Musser, $\mathrm{MD}, \mathrm{PhD}^{1,2}$
}

ов јестіvе. To assess the impact of Matrix-Assisted Laser Desorption/Ionization Time-of-Flight (MALDI-TOF) mass spectrometry for rapid pathogen identification directly from early-positive blood cultures coupled with an antimicrobial stewardship program (ASP) in two community hospitals. Process measures and outcomes prior and after implementation of MALDI-TOF/ASP were evaluated.

DESIGN. Multicenter retrospective study.

Setting. Two community hospitals in a system setting, Houston Methodist (HM) Sugar Land Hospital (235 beds) or HM Willowbrook Hospital (241 beds).

PAtients. Patients $\geq 18$ years of age with culture-proven Gram-negative bacteremia.

INTERVENTION. Blood cultures from both hospitals were sent to and processed at our central microbiology laboratory. Clinical pharmacists at respective hospitals were notified of pathogen ID and susceptibility results.

RESULTS. We evaluated 572 patients for possible inclusion. After pre-defined exclusion criteria, 151 patients were included in the pre-intervention group and 242 were included in the intervention group. After MALDI-TOF/ASP implementation, the mean identification time after culture positivity was significantly reduced from 32 hours ( \pm 16 hours) to 6.5 hours $( \pm 5.4$ hours $)(P<.001)$; mean time to susceptibility results was significantly reduced from $48( \pm 22)$ hours to $23( \pm 14)$ hours $(P<.001)$; and time to therapy adjustment was significantly reduced from $75( \pm 59)$ hours to $30( \pm 30)$ hours $(P<.001)$. Mean hospital costs per patient were $\$ 3,411$ less in the intervention group compared with the pre-intervention group $(\$ 18,645$ vs $\$ 15,234 ; P=.04)$.

CONCLUSION. This study is the first to analyze the impact of MALDI-TOF coupled with an ASP in a community hospital setting. Time to results significantly differed with the use of MALDI-TOF, and time to appropriate therapy was significantly improved with the addition of ASP.

Infect. Control Hosp. Epidemiol. 2016;37(4):425-432

Policy statements from the Society of Healthcare Epidemiology of America, Infectious Diseases Society of America, and Pediatric Infectious Diseases Society on antimicrobial stewardship programs (ASP) have recommended that the Centers for Medicare and Medicaid Services encourage healthcare institutions to develop and implement stewardship efforts. ${ }^{1}$ An optimal ASP is composed of a multidisciplinary team with dedicated oversight, policies, procedures, and accountability for reporting outcomes to continuously evaluate and promote optimal antimicrobial use. However, many community hospitals lack administrative or financial support, or the expertise, such as dedicated infectious diseases specialists, to successfully implement these strategies. ${ }^{1-4}$
While the impact of ASPs is often benchmarked on antimicrobial utilization data, disease state-driven approaches targeting timely antibiotic therapy for patients with bloodstream infections (BSIs) are essential. Delays in treatment have been associated with increased morbidity and mortality resulting in detrimental outcomes for patients. ${ }^{5,6}$ With mortality increasing by the hour for patients with BSI, reducing treatment time is essential to optimize outcomes. ${ }^{7}$ Novel approaches to ASP that have emphasized close collaboration between the microbiology laboratory and pharmacy have shown successful outcomes. These effects are more pronounced in patients with BSI where use of rapid diagnostics coupled with pharmacy intervention to improve antibiotic

Affiliations: 1. Houston Methodist Hospital System, Houston, Texas; 2. Houston Methodist Hospital System \& Houston Methodist Research Institute, Houston, Texas.

Received September 11, 2015; accepted November 20, 2015; electronically published January 7, 2016

(C) 2016 by The Society for Healthcare Epidemiology of America. All rights reserved. 0899-823X/2016/3704-0009. DOI: 10.1017/ice.2015.313 
optimization resulted in significant reductions in hospital length of stay (LOS), healthcare costs, and mortality. ${ }^{8-13}$

To date, the role of rapid diagnostics in ASPs has largely been studied in tertiary or quaternary care medical centers with dedicated infectious diseases pharmacists, complex patient populations, and robust clinical laboratory services. Thus, limited data are available in lower acuity community hospital settings. Subsequent to the success of the ASP at our quaternary-care flagship hospital with use of matrix-assisted laser desorption/ionization time-of-flight (MALDI-TOF) mass spectrometry and near real-time infectious diseases pharmacist notification, we endeavored to capitalize on the existing centralized clinical laboratory infrastructure and expertise described in previous publications and implement a proactive ASP in 2 representative community hospitals. ${ }^{8,9}$ In this study, the impact of rapid diagnostics with pharmacist intervention on patients with Gram-negative bacteremia is assessed in this community hospital setting.

\section{METHODS}

Houston Methodist (HM) consists of 7 hospitals: Houston Methodist Hospital, its flagship academic hospital in the Texas Medical Center (TMC) where clinical laboratory services are centralized, and 6 community hospitals throughout the Greater Houston metropolitan area. This study was comprised of 2 groups of patients $\geq 18$ years of age with Gram-negative bacteremia who were admitted to HM Sugar Land Hospital (235 beds, 20 miles from TMC) and HM Willowbrook Hospital (241 beds, 30 miles from TMC). A pre-intervention group was admitted between January 1, 2011, and December 31, 2011, and an intervention cohort was admitted between January 1, 2014, and December 31, 2014. The pre-intervention time frame was chosen because ASP efforts were not established nor was MALDI-TOF validated for routine identification (ID). A patient was excluded if his or her culture showed a pathogen that was not a facultative anaerobic or aerobic Gram-negative bacillus. Additionally, any polymicrobial bacteremia or subsequent bacteremia episodes after a subject's initial inclusion were not included in the analysis. Any patients who died prior to the blood culture becoming positive, and/or for whom criteria for discharge were determined by institutional policies rather than at the treating physician's discretion (ie, artificial life-sustaining device implantation, neurological complications, and/or elective procedures) were not included. This study was approved by the Institutional Review Board of HM Research Institute [IRB(2)1014-0219].

\section{Data Collection and Definitions}

Patients with Gram-negative BSIs were identified using Vigilanz, a real-time surveillance and notification software (Vigilanz, Minneapolis, MN). Data were collected on demographic characteristics, comorbidities, source and manifestations of BSI, microbiology, antibiotic therapy, and severity of illness by the Acute Physiology and Chronic Health Evaluation II (APACHE II) score. ${ }^{14,15}$ For the intervention time period, assessment of the clinical pharmacist's response to Vigilanz notifications were evaluated. BSI onset was defined as the time the first blood sample yielding the study isolate (index blood culture) was collected. Infection-related characteristics examined included infection source; pathogen species and susceptibility data; and time, dose, and route with antibiotics relative to time of index culture collection. The source of bacteremia was determined according to the definitions published by the Centers for Disease Control and Prevention. ${ }^{16}$ In each case, an effort was made to establish a primary source of infection.

We previously validated a method to identify Gram-negative bacteria directly from positive blood culture medium using MALDI-TOF Biotyper (Bruker Daltonics, Fremont, CA) and to rapidly perform susceptibility testing using the BD-Phoenix system in 2012. We have since successfully implemented in our clinical microbiology laboratory that serves as the reference laboratory for the HM system. ${ }^{17}$ Prior to using MALDI-TOF, positive blood culture specimens were inoculated on appropriate solid agar media and subsequently identified by conventional clinical microbiology procedures.

Prior to the implementation of ASP notification, Gramstain results were called to the nursing unit by the microbiology staff followed by passive reporting in the electronic medical record without further notification to the care team. Since the implementation of pharmacist intervention, Gramstain results have been reported to the nursing unit in a process identical to that in the pre-intervention group. Once the results become available, a page prompted by Vigilanz, is sent to the pharmacist on-call 24/7 with the organism ID and subsequent susceptibility profile. If necessary, the pharmacist contacts the treating provider to communicate the clinical interpretation of the results with recommendations for the most effective and targeted antimicrobial therapy via prospective audit and feedback strategy. Any decision to change antibiotic therapy remains at the discretion of the treating physician. Infectious disease pharmacists are available for consultation as needed and provide training to the on-call clinical pharmacists on the workflow process and interpretation of blood cultures and antibiotic selection.

In this study, therapy was appropriate when the administered regimen was active in vitro and, when available, was in accordance with current clinical guidelines regarding dosing and route of administration. ${ }^{9,18}$ Therapy was defined as inactive if the blood isolate was resistant to the agent(s) used or in the absence of any antibacterial medications. Empiric therapy was defined as antibiotics administered in the time period before the identification of the blood culture isolate and susceptibility results were available. De-escalation was defined as switching to a narrower-spectrum agent or decreasing the number of antibiotics from $\geq 2$ agents to a single agent when clinically appropriate. 
The duration of total hospital and ICU LOS were defined as by the difference in days between admission and discharge. Because death can artificially decrease LOS, LOS analyses were conducted with patients who survived to discharge. We used the American Hospital Association definition for a community and hospital system. ${ }^{19}$

Cost analysis was based on total hospital costs across all centers, including room and board, pharmacy, radiology, and laboratory. Cost data were obtained from an individual in the HM accounting department who was independent of the study team. All reported costs represent actual costs for the administration of patient care as determined by the individual departmental finance sections. ${ }^{8,9}$

Summary statistics for continuous variables were reported as mean \pm standard deviation (SD), and results for categorical variables were presented as frequencies. The MannWhitney test was employed to identify significantly different central locations between groups for continuously scaled variables, whereas the $\chi^{2}$ test was used to determine significantly different configurations across groups of categorical data. All tests were 2-tailed, and $P \leq .05$ represented statistical significance. $P$ values for the $\chi^{2}$ test were based on Fisher's exact test.

\section{RESULTS}

In total, 571 patients with Gram-negative BSIs were evaluated for inclusion. After exclusion criteria were applied, 390 patients were included in the final analysis. Of these, 149 patients comprised the pre-intervention group and 241 in the intervention group (Figure 1). Baseline demographics were similar between the 2 study groups with the exception of the proportion of patients with ICU admissions $(55.7 \%$ in the preintervention group vs $37.8 \%$ in the intervention group, $P<.001$ ) (Table 1). The most common infection site during both study periods was from genitourinary sources representing $48.3 \%$ in the pre-intervention group and $54.4 \%$ in the intervention group, followed by respiratory tract infections (11.4\% vs $12.5 \%$, respectively). Escherichia coli was the most frequently isolated pathogen, accounting for $53.7 \%$ in the pre-intervention group and $58.1 \%$ in the intervention group, followed by Klebsiella spp. (16.8\% vs $20.8 \%$, respectively). Both groups had similar frequencies of healthcare-associated and nosocomial infections.

The mean time to culture positivity was not significantly different between the pre-intervention and intervention periods (22 vs 19 hours, respectively). The mean time to pathogen

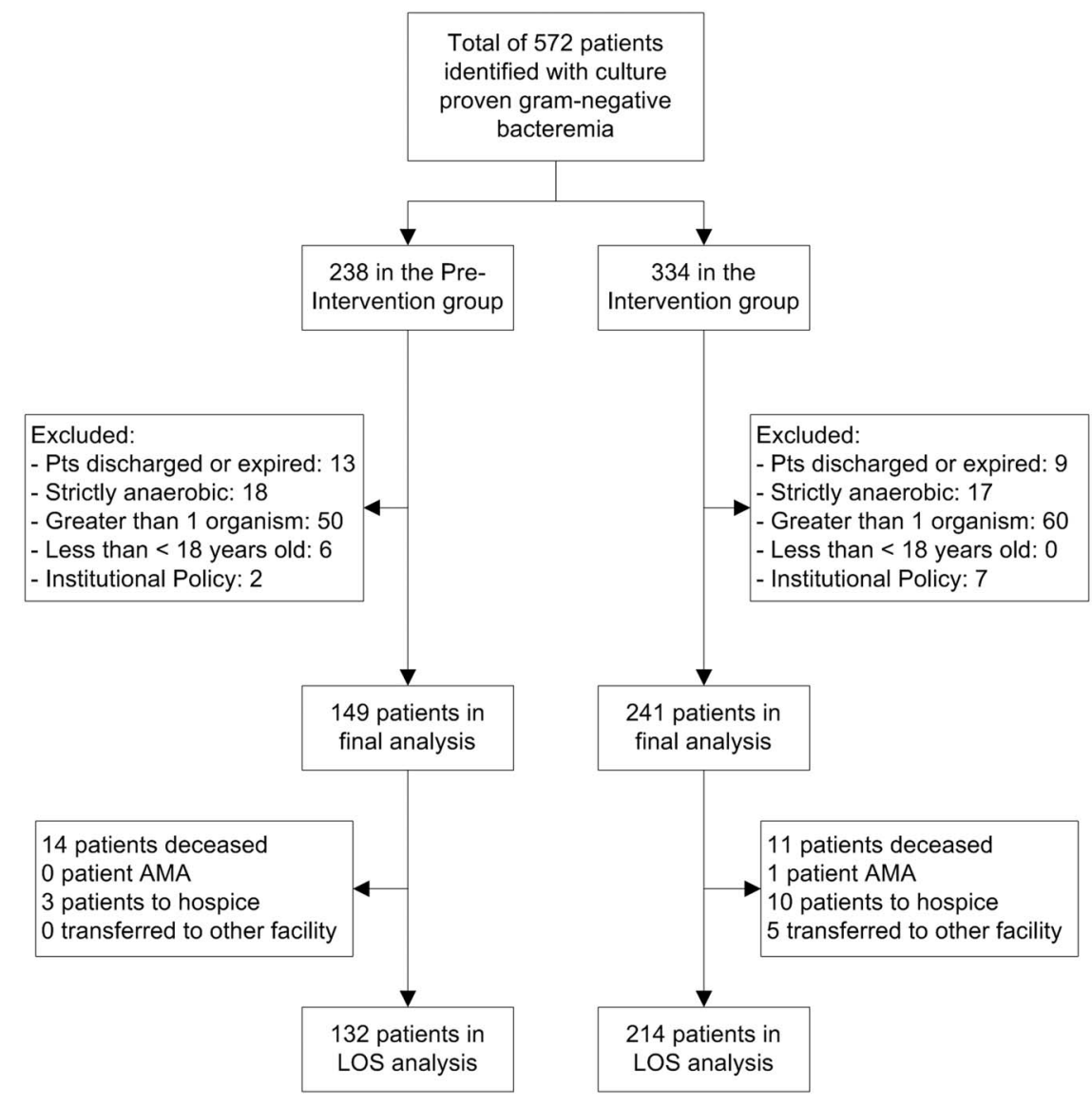

FIGURE 1. Eligibility and inclusion of study participants. Patients excluded due to being discharged or expired were those prior to time-to-positivity of index blood culture. AMA, against medical advice; LOS, length of stay. 
тавцE 1. Demographics and Baseline Characteristics ${ }^{\mathrm{a}}$

\begin{tabular}{|c|c|c|c|}
\hline Characteristic & Pre-intervention $(\mathrm{n}=149)$ & Intervention $(\mathrm{n}=241)$ & $P$ \\
\hline Age, $y$ & $66 \pm 16.8$ & $66.5 \pm 17.4$ & .8 \\
\hline Male sex, No. (\%) & $68(45.6)$ & $108(44.8)$ & .9 \\
\hline Body mass index, $\mathrm{kg} / \mathrm{m}^{2}$ & $27.5 \pm 6.6$ & $28.1 \pm 7.3$ & .8 \\
\hline APACHE II score ${ }^{b}$ & $16.6 \pm 7.7$ & $17.2 \pm 7.3$ & .4 \\
\hline ICU, No. $(\%)^{\mathrm{c}}$ & $83(55.7)$ & $91(37.8)$ & $<.001$ \\
\hline \multicolumn{4}{|l|}{ Pre-existing conditions, No. (\%) } \\
\hline Immunocompromised $^{\mathrm{d}}$ & $39(26.1)$ & $46(19.1)$ & .1 \\
\hline Organ transplant & $3(2)$ & $3(1.2)$ & .7 \\
\hline Malignancy & $12(8.1)$ & $22(9.1)$ & .9 \\
\hline Solid tumor & $11(7.4)$ & $19(7.9)$ & .9 \\
\hline Hematologic malignancy & $1(<1)$ & $3(1.2)$ & 1 \\
\hline Chronic lung disease & $8(5.4)$ & $20(8.3)$ & .3 \\
\hline Cardiovascular disease & $105(70.5)$ & $183(75.9)$ & .2 \\
\hline Cerebrovascular disease & $19(12.8)$ & $26(10.8)$ & .6 \\
\hline Diabetes & $47(31.5)$ & $81(33.6)$ & .7 \\
\hline Chronic kidney disease & $11(7.4)$ & $16(6.6)$ & .8 \\
\hline End-stage renal disease & $15(10.1)$ & $18(7.5)$ & .5 \\
\hline Liver disease & $7(4.7)$ & $14(5.8)$ & .8 \\
\hline \multicolumn{4}{|l|}{ Infection source, No. (\%) } \\
\hline Genitourinary & $72(48.3)$ & $131(54.4)$ & .3 \\
\hline Respiratory & $17(11.4)$ & $30(12.5)$ & .9 \\
\hline Intra-abdominal & $13(8.7)$ & $24(10)$ & .7 \\
\hline Surgical site or skin structure & $7(4.7)$ & $7(2.9)$ & .4 \\
\hline Line infection & $11(7.4)$ & $17(7.1)$ & 1 \\
\hline Febrile neutropenia & $7(4.7)$ & $6(2.5)$ & .3 \\
\hline Other & $6(4)$ & $4(1.7)$ & .2 \\
\hline$>1$ source & $2(1.3)$ & $7(2.9)$ & .5 \\
\hline Unidentified & $14(9.4)$ & $15(6.2)$ & .3 \\
\hline \multicolumn{4}{|l|}{ Organism, No. (\%) } \\
\hline Escherichia spp. & $80(53.7)$ & $140(58.1)$ & .4 \\
\hline Klebsiella spp. & $25(16.8)$ & $50(20.8)$ & .4 \\
\hline Pseudomonas spp. & $10(6.7)$ & $16(6.6)$ & 1 \\
\hline Proteus spp. & $11(7.4)$ & $12(5)$ & .3 \\
\hline Other & $23(8.1)$ & $23(9.5)$ & .1 \\
\hline MDRO/ESBL, No. (\%) & $17(11.4)$ & $29(10)$ & .9 \\
\hline Healthcare-associated infection, No. $(\%)^{\mathrm{e}}$ & $81(54.4)$ & $114(47.3)$ & .2 \\
\hline Nosocomial $(\%)^{\mathrm{f}}$ & $14(9.4)$ & $10(4.2)$ & .06 \\
\hline Pre-infection LOS, d, median (IQR) ${ }^{\mathrm{g}}$ & $5.4(4.4-8.3)$ & $4.8(3-5.9)$ & .3 \\
\hline ICU patients not on active therapy at TTP $(\%)^{\mathrm{c}}$ & $13(48.1)$ & $17(35.4)$ & .3 \\
\hline
\end{tabular}

NOTE. APACHE II, Acute Physiology and Chronic Health Evaluation II; HIV/AIDS, human immunodeficiency virus/acquired immunodeficiency syndrome; IQR, interquartile range; LOS, length of stay; MDRO/ESBL, multidrug-resistant organism/extended-spectrum beta-lactamase-producing organism; TTP, time to positivity.

${ }^{\text {a }}$ Plus-minus values are means \pm SDs.

${ }^{b}$ The APACHE II score was calculated for ICU patients based on clinical data present during the 24 hours preceding the index blood culture. Missing variables were assumed to be normal.

'Includes patients with an intensive care unit admission at any time during the index hospitalization.

${ }^{\mathrm{d}}$ Immunosuppressive therapy was defined as receipt of cytotoxic agents within 6 weeks, corticosteroids at a dosage of $15 \mathrm{mg}$ or more of prednisolone (or equivalent) daily for longer than 1 week within 4 weeks, or other immunosuppressive agents within 2 weeks before bacteremia onset.

${ }^{\mathrm{e}}$ Includes patients with recent contact with the healthcare system: recipients of recent intravenous therapy, dialysis, or home wound care; residence at long-term care facilities; and recent hospitalizations. ${ }^{15}$

${ }^{\mathrm{f}}$ Limited to patients hospitalized for $\geq 48$ hours prior to collection of the index culture (onset of bloodstream infection).

gre-infection LOS limited to patients with nosocomial acquisition of bloodstream infection and who survived to hospital discharge (pre-intervention group $[n=14]$ and intervention group $[n=10]$ ). 
identification was significantly reduced after MALDI-TOF/ ASP implementation, from 32 hours ( \pm 16 hours) to 6.5 hours ( \pm 5.4 hours) $(P<.001)$; mean time to antibiotic susceptibility results was significantly reduced from $48( \pm 22)$ to $22( \pm 14)$ hours $(P<.001)$; and time to therapy adjustment was significantly reduced from $71( \pm 59)$ hours to $30( \pm 30)$ hours $(P<.001)$. Therapy adjustment included de-escalation and/or escalation of antibiotic therapy, dosing and/or administration route modifications, and/or discontinuation of unnecessary Gram-positive coverage.

Clinical pharmacists made 120 recommendations to providers during the intervention period. Of those, $65 \%(n=79)$ were accepted (Table 2). Overall, the proportion of patients whose antibiotic regimen was adjusted was significantly increased from $63.8 \%$ in the pre-intervention group to $84.2 \%$ in the intervention group $(P<.001)$. Additionally, the time to therapy adjustment was significantly decreased from an average of 71 hours in the pre-intervention group to 30 hours in the intervention group $(P<.001)$.

The hospital LOS in survivors from infection onset did not differ significantly between the study groups with an average of $6.4 \pm 3.8$ days (range, 0.8-24 days) in both groups and $3.7 \pm 3.4$ days (range, $0.02-21.8$ days) for ICU LOS (Table 3). Overall mortality did not differ significantly between the groups $(9.4 \%$ vs $4.9 \%, P=.07)$.

TABLE 2. Clinical Pharmacist Interventions

\begin{tabular}{lcc}
\hline Intervention Type & Accepted & Rejected \\
\hline Escalation, No. (\%) & $22(18.3)$ & $4(3.3)$ \\
Selection, No. (\%) & $20(16.7)$ & $4(3.3)$ \\
Deescalation, No. (\%) & $37(30.8)$ & $33(27.5)$ \\
\hline
\end{tabular}

Similar proportions of patients lacked active antibiotic therapy at culture positivity (27 of 149 [18\%] pre-intervention and 48 of 241 [20\%] intervention; Figure 2). The time to active treatment was significantly shorter in the intervention cohort. At 24 hours, time to active treatment was $8 \%$ shorter in the pre-intervention group vs $3 \%$ shorter in the intervention group with inactive therapy $(P=.01)$. At 48 hours, time to active treatment was $4 \%$ shorter in the pre-intervention group vs $<1 \%$ shorter in the intervention group with inactive therapy $(P=.008)$. At 72 hours, time to active treatment was $3 \%$ shorter in the pre-intervention group and was no shorter in the intervention group with inactive therapy $(P=.01)$. Mortality rates were significantly reduced from $25 \%(\mathrm{n}=7$ of 27$)$ in the pre-intervention group to $2.1 \%$ in the intervention group $(\mathrm{n}=1$ of 48$)(P=.006)$.

Mean hospital costs per patient were $\$ 3,411$ less in the intervention group than in the pre-intervention group $(\$ 18,645$ vs $\$ 15,234 ; P=.04)$. Costs decreased in patients without ICU admissions ( $\$ 11,930$ vs $\$ 10,842 ; P=.3$ ) and ICU patients ( $\$ 24,116$ vs $\$ 22,473 ; P=.7$ ) although this decrease did not reach statistical significance. Costs were higher among patients without active empiric therapy ( $\$ 26,462$ vs $\$ 19,002$; $P=.5$ ) (Table 3).

\section{DISCUSSION}

We evaluated the impact of rapid diagnostics with an active pharmacy intervention on patients with Gram-negative bacteremia in a community hospital setting and showed favorable outcomes. Significant improvements were achieved in time to infecting pathogen ID, antimicrobial susceptibilities, and overall management of antibiotic therapy. Importantly, a

TABle 3. Patient Outcomes

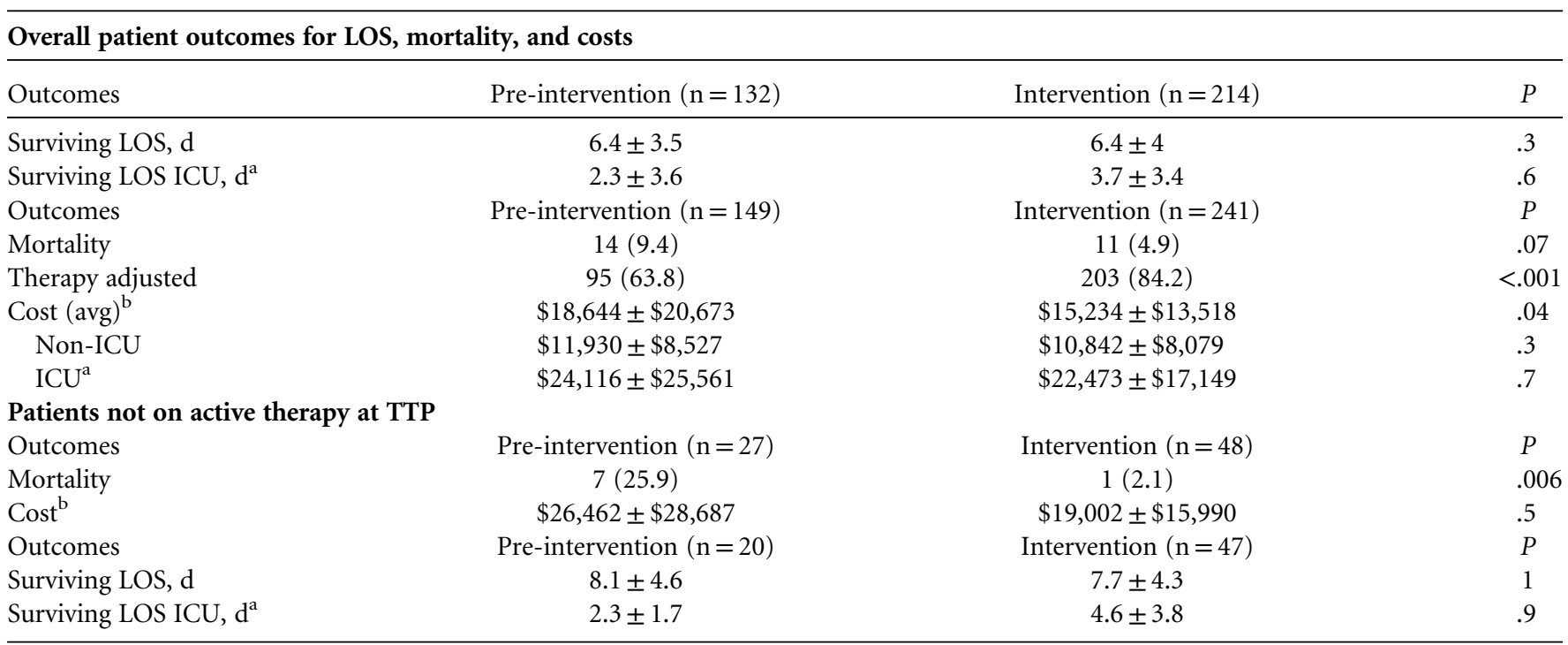

NOTE. LOS, length of stay; ICU, intensive care unit; TTP, time to positivity; Avg, average; Pts, patients.

${ }^{a}$ Patients in ICU group included any admissions to the ICU at any point within hospitalization.

${ }^{\mathrm{b}}$ Costs in the pre-intervention group were adjusted to comparable dollars. ${ }^{8,9}$ 


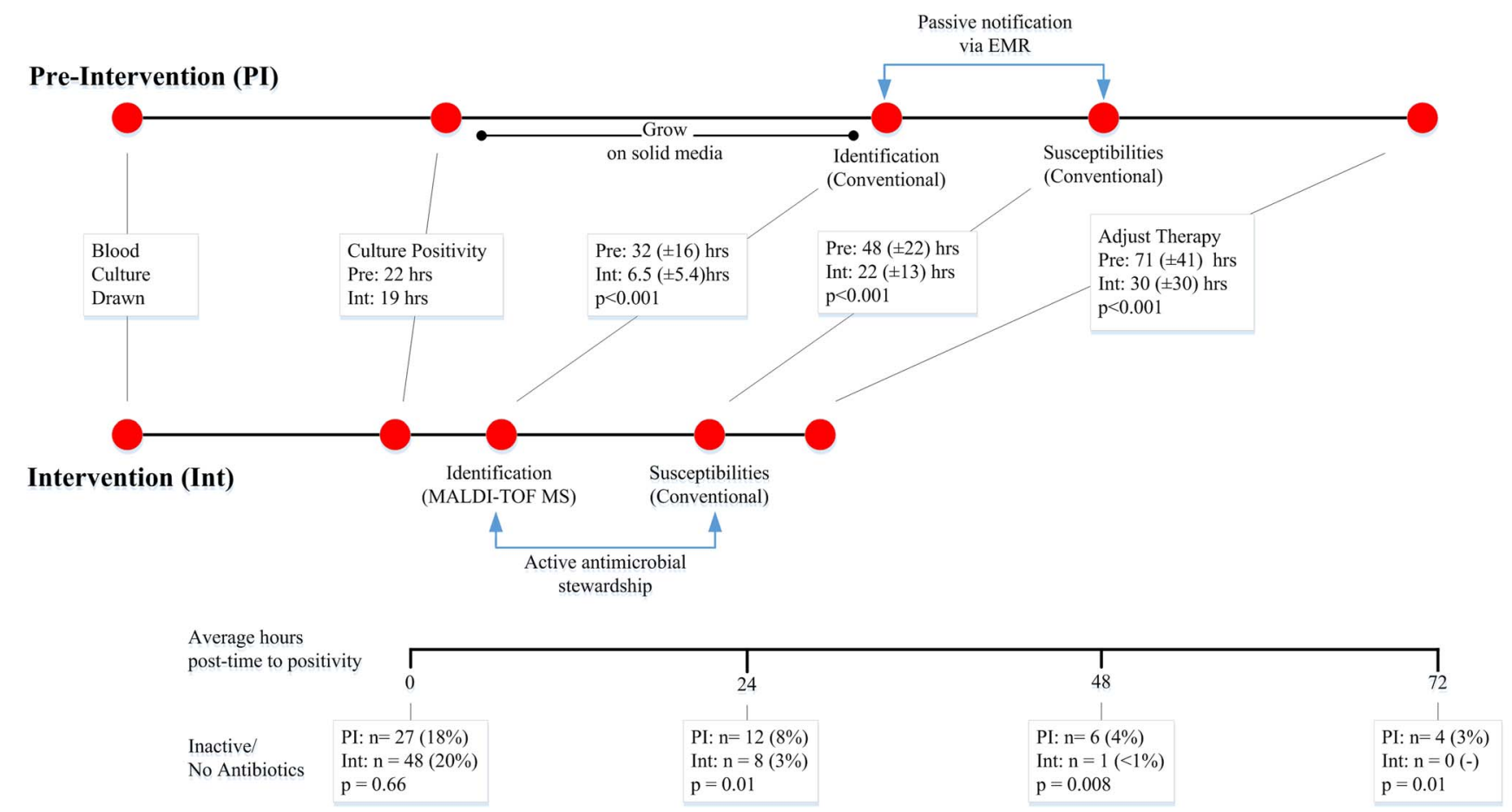

FIGURE 2. Timeline comparison of pre-intervention and intervention illustrating difference in laboratory, reporting, and interventions. Adjusted therapy includes de-escalation/escalation of antibiotic therapy, dosing/route modifications, and/or discontinuation of unnecessary Gram-positive coverage. Boxes represent average time in hours until corresponding information reported or adjustment of therapy. The bottom horizontal line represents the global study/patient timeline (hours) and includes point measurements (below) for patients on inactive therapy at 0,24 , and 48 hours in both groups. EMR, electronic medical record; MALDI-TOF, matrix-assisted laser desorption and ionization time-of-flight mass spectrometry.

significant mortality benefit was observed among patients on inactive empiric treatment (25\% in the pre-intervention group vs $2.1 \%$ in the intervention group; $P=.006$ ). These findings are consistent with previous studies describing the increased morbidity and mortality among patients with delays in appropriate antibiotic therapy; they further highlight the importance of real-time antimicrobial stewardship evaluation and use of rapid diagnostics. ${ }^{5-7}$ In our previous studies by Perez et al, ${ }^{8}$ MALDI-TOF was utilized for rapid pathogen ID in addition to real-time pharmacy intervention at a tertiary care referral center. The results of this study demonstrated significant decreases in (1) length of hospitalization from 11.9 days (pre-intervention group) to 9.3 days (intervention group), (2) 30-day all-cause mortality for the intervention group, and (3) mean cost per patient, witha decrease of $\$ 19,547$.

In this study, we aimed to determine whether integrating rapid diagnostics with antimicrobial stewardship would improve outcomes in hospitalized patients in a community hospital setting that lacks pharmacists trained in infectious diseases. Our findings illustrate that with training, education, and availability of an infectious diseases pharmacist consultation, generalist clinical pharmacists can successfully assist in the notification and interpretation of culture results in a setting that applies to the majority of hospital facilities nationwide. ${ }^{19}$ Despite barriers in community hospitals, ASPs have developed in many forms and have prospered with use of traditional interventions such as intravenous-to-oral conversions, educational programs, daily review of antimicrobial orders by a pharmacist, drug audits with feedback, and prior authorizations and/or restrictions. ${ }^{2-4}$ These simple and effective interventions have been shown to improve patient outcomes, to reduce costs, and to decrease the development of antimicrobial resistance. ${ }^{2-4}$ To our knowledge, only 1 study to date has evaluated the use of rapid diagnostics in a community hospital setting using the Nanosphere Verigene test to evaluate Grampositive bacteremia in conjunction with ASP. Box et $\mathrm{al}^{20}$ illustrated a similar decrease in time to therapy adjustment along with decreased LOS and hospital costs.

The relationship between prompt, appropriate initiation of antibiotic therapy and improved patient care confirms the importance of implementing rapid diagnostics in the community setting. Collaboration between the microbiology laboratory and pharmacy facilitated an expedited distribution of clinically actionable information to significantly improve outcomes. However, we did not observe a LOS reduction as we did in our previous studies conducted in a quaternary-care setting. ${ }^{8}$ We did find many of the same improvements in 
process outcomes and therapeutic benefits in a very different patient population. When comparing baseline patient characteristics across studies, the most striking difference is that the lower patient acuity among community hospitals accounts for the much shorter overall LOS seen during both study periods (LOS, 6.5 days). Patients in the community hospital setting were less likely to be transplant recipients, immunocompromised, or have malignancies than patients in the quaternarycare setting. Additionally, the underlying source of BSI was markedly different; there were more genitourinary sources in the community hospitals and far fewer indwelling vascular devices. These findings are consistent with patient characteristics found in publications describing infectious etiologies among patients in community hospitals. ${ }^{21}$

Even with lower patient acuity, we observed an overall lower inpatient mortality rate for the intervention group that was not statistically significant $(9.4 \%$ vs $4.9 \% ; P=.07)$. Despite a similar and sizeable proportion of patients who were not receiving active antibiotic therapy at the time to positivity (TTP) during both study periods ( $18 \%$ and $20 \% ; P=.07)$, we found a clear benefit to patients: the time to active therapy was significantly reduced during the intervention study period (Figure 2). The decreased time to active therapy was associated with a mortality benefit among those patients with less delay (25\% in the pre-intervention group vs $2.1 \%$ in the intervention group; $P=.006)$. Furthermore, there was a nonsignificant decrease of $\$ 7,470$ per hospitalization among these patients $(P=.5)$ leading to a projected estimated savings of $\$ 360,000$ for the intervention cohort. Notably, no difference was observed in the number of patients admitted to the ICU in this subset of patients.

In this study, we identified a significant difference in hospital costs that favored the intervention group by an overall average of $\$ 3,411$ per patient cost savings. Although the exact reason for the cost savings remains unclear, a likely source is the greater number of patients with ICU admissions in the pre-intervention cohort. Because patients with ICU admissions inherently incur higher healthcare costs, we evaluated the groups separately (non-ICU patients and ICU patients). We found that a cost savings was maintained outside of ICU admissions for the intervention cohort, though the sample size was smaller and the difference did not reachstatistical significance.

Finally, in this study, we demonstrated the feasibility and utility of having a centralized clinical microbiology laboratory located in a metropolitan area within 30 miles of several facilities. This laboratory placement could potentially serve as a model for the existing 3,144 community hospitals across the United States where it may not be possible or cost-effective to have laboratories equipped with rapid diagnostics at in-house locations. ${ }^{19}$

We are aware of several limitations to this study. First, this study took place in 2 community hospitals; its generalizability may be limited to similar system community settings. The patient data were analyzed retrospectively, which allows the possibility of information bias. Physician prescribing practice variations between sites, over time, and training/experience were not examined. In the analysis, we did not compare the impact of rapid diagnostics without ASP or vice versa, making it difficult to assess the individual impact of these elements. However, our objective was to evaluate the effect of the combined intervention. Finally, the clinical pharmacists responding to BSI notifications were not formally trained in infectious diseases. The use of general clinical pharmacists does make the study more generalizable; not all community hospitals have an infectious diseases trained pharmacist on their staff. Although this may be a disadvantage for the study, it highlights the necessity of trained infectious diseases pharmacists in the community setting. Perez et $\mathrm{al}^{8}$ found a recommendation acceptance rate of $91 \%$ when infectious diseases pharmacists intervened, compared with $65 \%$ acceptance in our study. Reasons for the differences are unclear and will be a focus of future efforts. ASP teams staffed by dedicated infectious diseases pharmacists are associated with better adherence to evidence-based antibiotic prescribing measures, including selection, de-escalation, modification at 24 hours, etc. This area requires further study to assess the impact these individuals would have to a program with rapid diagnostics.

In the growing use of rapid diagnostics, many studies have shown the benefit of their use in the treatment of BSI in tertiary and quaternary settings even though the evidence is lacking in the community setting. Our results demonstrate the benefit of rapid diagnostics coupled with ASP on time to therapy adjustment; the impact is greatest on patients who did not have active therapy at the time of culture positivity and on their clinical outcomes. The use of rapid diagnostics coupled with antimicrobial stewardship should be incorporated in the community setting to improve time to therapy adjustment in patients with Gram-negative bacteremia. Our data unambiguously demonstrate improved patient outcomes, most notably for those who lack active therapy.

\section{ACKNOWLEDGMENTS}

We thank the Clinical Microbiology Laboratory staff and the Clinical Pharmacy Services for assistance with the study. We thank James R. Davis, $\mathrm{PhD}$, Department of Pathology and Genomic Medicine, $\mathrm{HMH}$, for advice and assistance. We gratefully acknowledge K. E. Stockbauer, PhD, Office of Academic Development, Department of Pathology and Genomic Medicine, $\mathrm{HMH}$, for assistance with manuscript preparation.

Financial support. No financial support was provided relevant to this article.

Potential conflicts of interest. All authors report no conflicts of interest relevant to this article.

Address correspondence to Katherine K. Perez, 6565 Fannin, DB1-09, Houston, Texas 77030 (kkperez@houstonmethodist.org).

\section{REFERENCES}

1. Policy Statement on Antimicrobial Stewardship by the Society for Healthcare Epidemiology of America (SHEA), the Infectious Diseases Society of America (IDSA), and the Pediatric Infectious Diseases Society (PIDS). Infect Control Hosp Epidemiol 2012;33:322-327. 
2. Trivedi KK, Kuper K. Hospital antimicrobial stewardship in the nonuniversity setting. Infect Dis Clin N Am 2014;28:281-289.

3. Ohl CA, Dodds Ashley ES. Antimicrobial stewardship programs in community hospitals: The evidence base and case studies. Clin Infect Dis 2011;53:S23-S28.

4. Bartlett JM, Siola PL. Implementation and first-year results of an antimicrobial stewardship program at a community hospital. Am J Health-Syst Pharm 2014;71:943-949.

5. Ibrahim EH, Sherman G, Ward S, Fraser VJ, Kollef MH. The influence of inadequate antimicrobial treatment of bloodstream infections on patient outcomes in the ICU setting. Chest 2000;118:146-155.

6. Lodise TP, McKinnon PS, Swiderski L, Rybak MJ. Outcomes analysis of delayed antibiotic treatment for hospital-acquired Staphylococcus aureus bacteremia. Clin Infect Dis 2003;36: 1418-1423.

7. Kumar A, Roberts D, Wood KE, et al. Duration of hypotension before initiation of effective antimicrobial therapy is the critical determinant of survival in human septic shock. Crit Care Med 2006;34:1589-1596.

8. Perez KK, Olsen RJ, Musick WL, Cernoch PL, Davis JR, Peterson LE, Musser JM. Integrating rapid diagnostics and antimicrobial stewardship improves outcomes in patients with antibiotic-resistant Gram-negative bacteremia. J Infect 2014; 69:216-225.

9. Perez KK, Olsen RJ, Musick WL, et al. Integrating rapid pathogen identification and antimicrobial stewardship significantly decreases hospital costs. Arch Pathol Lab Med 2013;137: 1247-1254.

10. Bauer KA, West JE, Balada-Llasat JM, Pancholi P, Stevenson KB, Goff DA. An antimicrobial stewardship program's impact with rapid polymerase chain reaction methicillin-resistant Staphylococcus aureus/S. aureus blood culture test in patients with S. aureus bacteremia. Clin Infect Dis 2010;51:1074-1080.

11. Huang AM, Newton D, Kunapuli A, et al. Impact of rapid organism identification via matrix assisted laser desorption/ ionization time-of-flight combined with antimicrobial stewardship team intervention in adult patients with bacteremia and candidemia. Clin Infect Dis 2013;57:1237-1245.
12. Heil EL, Daniels LM, Long DM, Rodino KG, Weber DJ, Miller MB. Impact of a rapid peptide nucleic acid fluorescence in situ hybridization assay on treatment of Candida infections. Am J Health Syst Pharm 2012;69:1910-1914.

13. Vlek AL, Bonten MJ, Boel CH. Direct matrix-assisted laser desorption ionization time-of-flight mass spectrometry improves appropriateness of antibiotic treatment of bacteremia. PLoS One 2012;7:e32589.

14. Knaus WA, Draper EA, Wagner DP, Zimmerman JE. APACHE II: a severity of disease classification system. Crit Care Med 1985;13:818-829.

15. Guidelinesfor the management of adults with hospital-acquired, ventilator-associated, and healthcare-associated pneumonia. Am J Respir Crit Care Med 2005;171:388-416.

16. Garner JS, Jarvis WR, Emori TG, Horan TC, Hughes JM. CDC definitions for nosocomial infections, 1988. Am J Infect Control 1988;16:128-140.

17. Wimmer JL, Long SW, Cernoch P, et al. Strategy for rapid identification and antibiotic susceptibility testing of Gram-negative bacteria directly recovered from positive blood cultures using the Bruker MALDI Biotyper and the BD Phoenix system. J Clin Microbiol 2012;50:2452-2454.

18. McGregor JC, Rich SE, Harris AD, et al. A systematic review of the methods used to assess the association between appropriate antibiotic therapy and mortality in bacteremic patients. Clin Infect Dis 2007;45:329-337.

19. Facts on US Hospitals. American Hospital Association website. http://www.aha.org/research/rc/stat-studies/fast-facts.shtml. Published 2015. Accessed August 18, 2015.

20. Box MJ, Sullivan EL, Ortwine KN, et al. Outcomes of rapid identification for Gram-positive bacteremia in combination with antibiotic stewardship at a community-based hospital system. Pharmacotherapy 2015;35:269-276.

21. Rodríguez-Baño J, López-Prieto $\mathrm{MD}$, Portillo $\mathrm{MM}$, et al. Epidemiology and clinical features of community-acquired, healthcare-associated and nosocomial bloodstream infections in tertiary-care and community hospitals. Clin Microbiol Infect 2010;16:1408-1413. 